\title{
Reinforcement ropes against shear in leaves
}

\author{
C. Mattheck, A. Sauer \& R. Kappel \\ Institute for Materials Research II, \\ Forschungszentrum Karlsruhe GmbH, Germany
}

\begin{abstract}
Biological structures consist of mechanical load carriers, which are highly optimized in terms of mechanical strength and minimum weight. Many parts of these structures act as tension ropes, even if this cannot always be identified immediately. The advantage of tension-loaded components is that they cannot fail by kinking or buckling like pressure-loaded ones if their aspect ratio is too high.

Special application ranges for tension ropes exist in structures, which are frequently exposed to shear loading. Examples are leaves in the wind or bird feathers during a flight. When looking at the supporting skeletons, there are tensile ropes releasing the structure from shear.

To verify this statement, the vein pattern of a leaf is studied for mechanical suitability using the Soft Kill Option (SKO) structure optimization program. It is found that the leaf is formed in a mechanically optimum manner. The structure is stiffened by tension ropes acting as shear killers. Subsequently, an SKO parameter study is performed to investigate and determine influencing parameters and their relevance to the formation of shear killers.

Keywords: leaf structure, shear killer, bionic, biomimetic, vein angle.
\end{abstract}

\section{Introduction}

When a storm shakes and bends a tree, enormous forces act on the leaves. The leaf surface with its side veins that branch off at about $45^{\circ}$ resists these wind forces. Side veins transmit the tension induced by the wind from the leaf surface via the main vein into the branch and, hence, act like stiffening tension ropes. Their efficiency is reflected by the fact that in reality a storm will more likely tear the leaves off the stalks than rip off the leaf surface. 


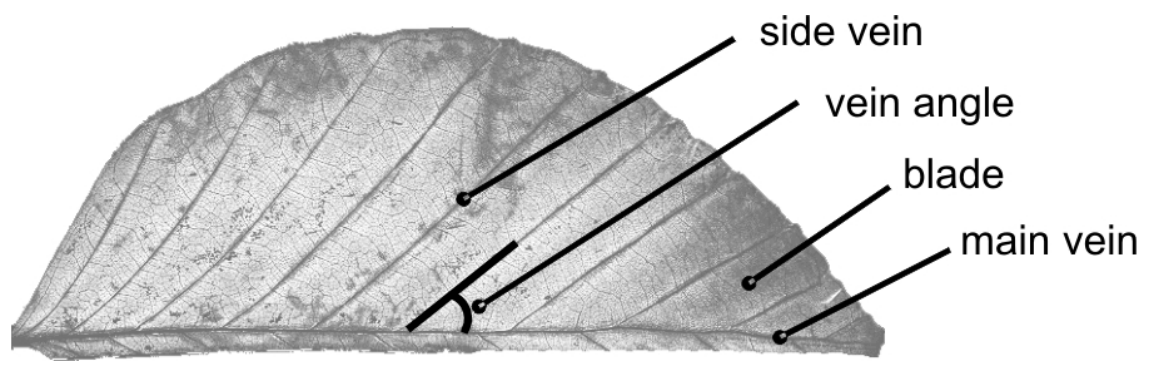

Figure 1: $\quad$ Leaf elements of a European Beech (Fagus silvatica).

Leaves wave like flags in the wind, which is why the wind force nearly always acts parallel to the main vein, Fig. 2. Longitudinal shear stresses prevent the leaf halves from shearing off the main vein. Symmetry of the stress tensor, see [2], requires equal lateral shear stresses that form the shear square together with the longitudinal shear stresses.

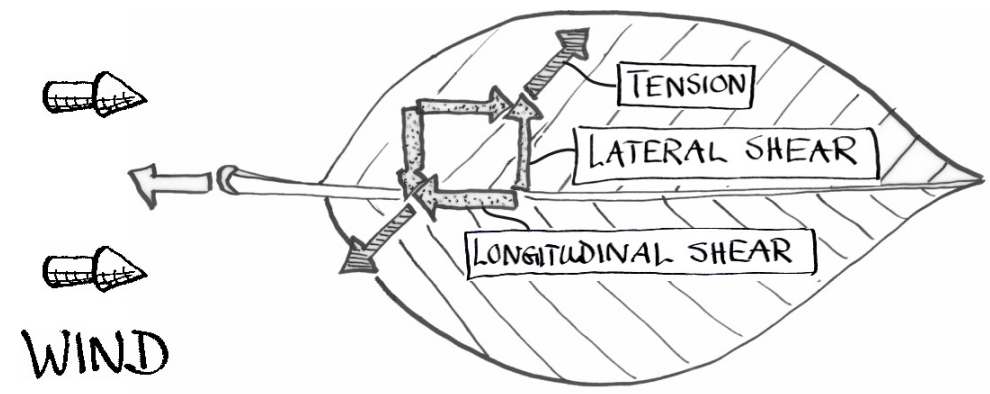

Figure 2: $\quad 45^{\circ}$ side vein angle with the shear square.

The direction of the resulting tension force that is equivalent to the shear forces may be determined by vector addition of a longitudinal and a lateral shear arrow and, hence, is offset by $45^{\circ}$.

In [3], the $45^{\circ}$ orientation of the leaves' side veins is explained theoretically with the help of the shear square. For verification, the SKO method (Soft Kill Option that simulates the osteoclasts in the living bone) will be used in this study.

\section{FEM analogous model}

To determine the loads of a leaf by means of FEM (finite-element method), a calculation model is needed, which is reduced to mechanically important properties. As the leaf is symmetrical to the center line, only half of a leaf is required by the model. As a result, calculation expenditure is also halved. The simplified setup of this leaf half, Fig. 3, resembles a layered cake and consists of a blade layer and a side vein layer on top. Both layers are firmly connected with 
each other, each single layer being divided into numerous finite elements, which is shown schematically by the leaf cross section. The view from above additionally shows the homogeneous surface load caused by the wind and the leaf's fixation at the symmetry line (main vein).

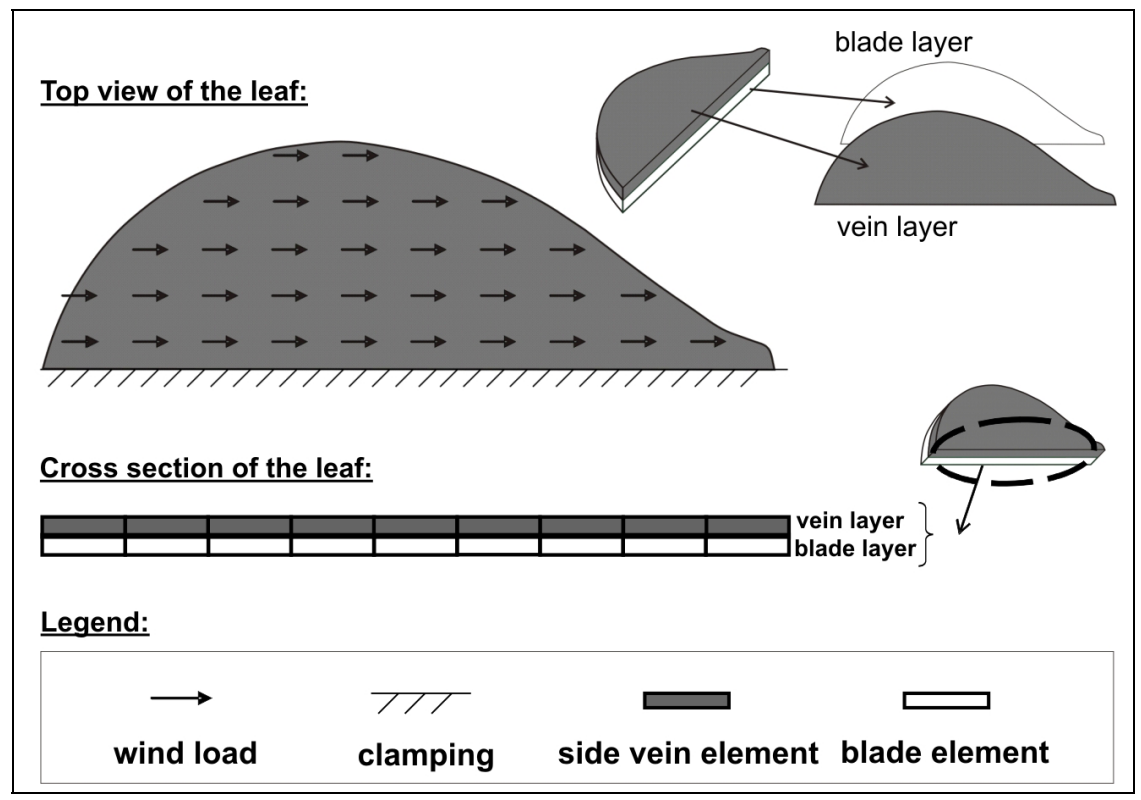

Figure 3: View from above and cross section of the finite-element analogous model.

\section{SKO}

The SKO method simulates adaptive mineralization processes in the living bone. Higher loaded areas are stiffened, less loaded areas are softened and eventually removed. In this way, the stability required is reached with a minimum use of material.

In this SKO optimization, the blade layer (light grey) is not changed. However, the load of the individual blade elements is determined. Depending on the element load, the following cases are distinguished and pictured in Fig. 4:

1. If the element load is smaller than a given reference stress, loadability of the blade is sufficient, nothing happens.

2. If the load of the blade element exceeds the reference stress, however, the leaf area is overloaded. In this case, the overloaded blade is reinforced by additional side vein elements and, hence, loadability is increased.

The result of a SKO calculation is displayed in Fig. 5. One can see how slender, triangle-shaped side veins develop from the center line towards the edge of the leaf in the formerly homogeneous side vein layer (dark grey). At the leaf edge and leaf tip, no side veins exist. In these areas, load remains below the 
reference stress, as a result of which all side vein elements are removed by the SKO method and only the blade remains. This area is sufficient to take up the local forces.

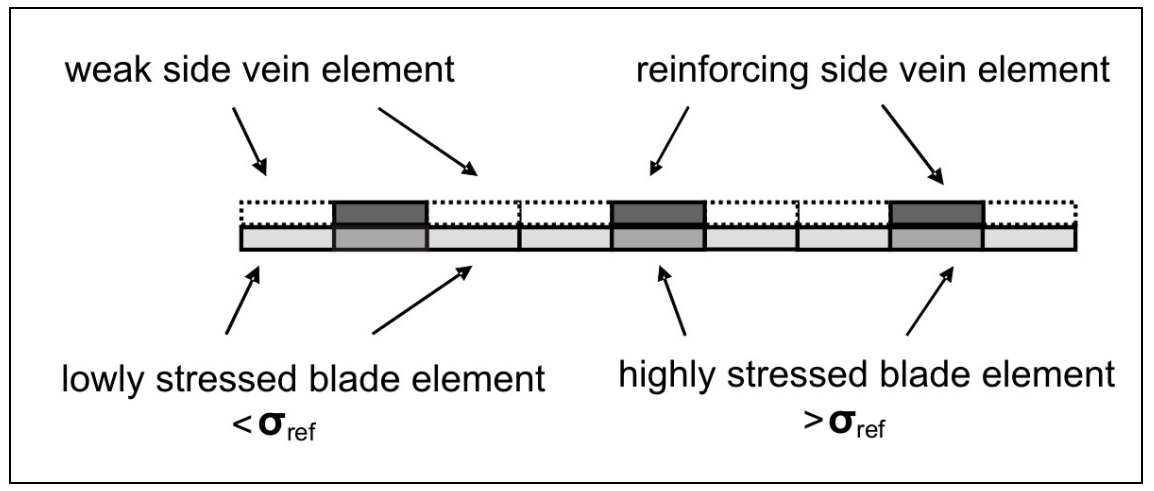

Figure 4: Principle of the SKO method.

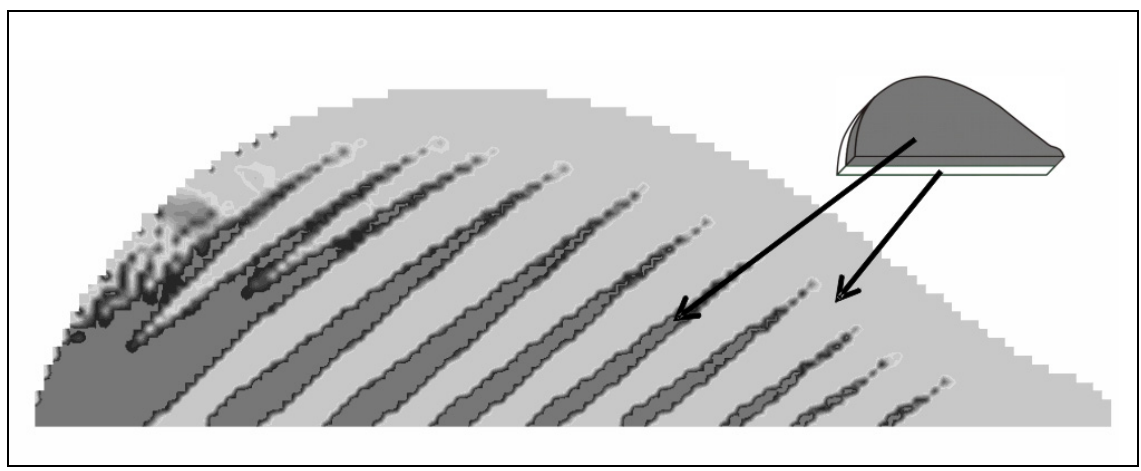

Figure 5: $\quad$ Principle representation of an SKO result.

\section{Parameter study}

In the following Figs. 6 and 7, the factors influencing side vein formation shall be studied in more details.

In Fig. 6, the reference stress of the blade layer is varied at the same surface load. The amount of this reference stress influences the development and number of side veins. In Fig. 7, the influence of the side "vein: blade" stiffness ratio on the side vein angle is plotted at constant load.

By changing the reference stress of the leaf blade, i.e. its permissible operation load, the number of side veins and their size are affected among others. A weak leaf blade (Fig. 6A) needs many side veins as helpers (shear killers) which are even connected to each other. A leaf blade with a higher loading 
capacity transfers more forces and requires less stiffening side veins. Hence, the leaf with the highest loadable blade (Fig. 6D) has the least side veins.

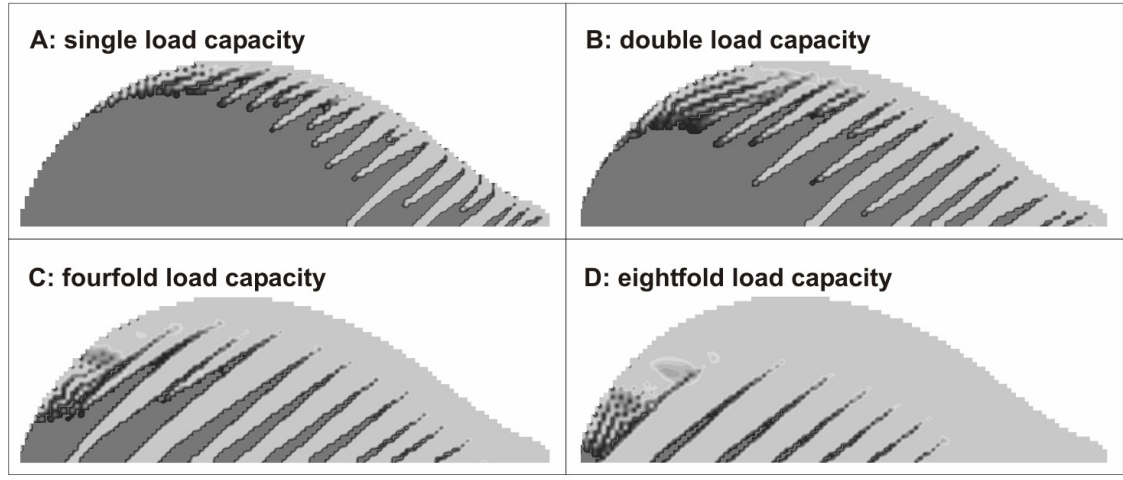

Figure 6: Influence of the reference stress on the formation of side veins.

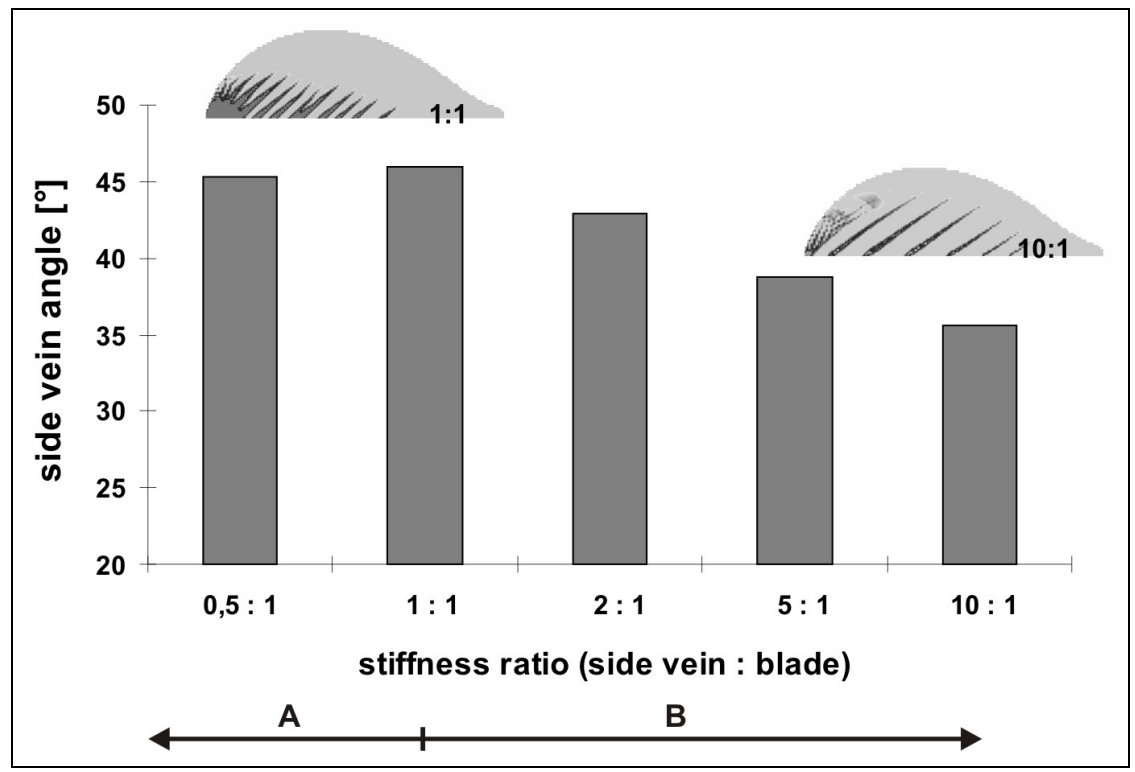

Figure 7: Influence of the stiffness ratio between side vein layer and leaf blade layer on the side vein angle.

When the stiffness of the side vein layer is increased in relation to the blade layer, the side vein angle is affected only slightly, but continuously. When changing the stiffness ratio by a factor of 20 , the angle changes by just $10^{\circ}$.

The following two cases can be distinguished:

A. The side vein angle amounts to $45^{\circ}$ 
The blade is as stiff as or stiffer than the side vein.

B. The angle decreases

The side vein is stiffer than the blade. With increasing stiffness of the side vein, the optimum branching-off angle of $45^{\circ}$ decreases slightly. In the extreme case (theoretical), the blade is so soft that it can no longer take up any pressure. In this case, the side veins will arrange along the main vein like brush hair.

\section{Summary}

With the help of the shear square, a side vein angle of appx. $45^{\circ}$ is obtained for leaves with a homogeneous structure. Some natural leaves, however, exhibit an angle other than these $45^{\circ}$. This may be due to the fact that the side veins fulfil various functions at the same time, e.g. transport function and transpiration. This study is restricted to the mechanical property exclusively.

To study the mechanically optimum side vein angle, an FEM-supported parameter study is carried out. This study confirms that the optimum side vein angle is around $45^{\circ}$. It also becomes obvious that functions in certain areas are separated between tension- and pressure-transferring structural elements. The side vein transfers tension only, whereas the blade mainly transfers pressure.

Strength of the blade influences the side vein formation. A blade with a higher loadability needs less and smaller side veins than a weaker blade. The side vein angle is influenced by the stiffness ratio between side vein and blade. At the same stiffness ratio, the mechanically optimum angle amounts to $45^{\circ}$. If the side vein stiffness increases, however, the side vein angle decreases slightly, but continuously.

\section{Conclusion}

The SKO method that simulates bone growth may also be applied to leaves, as is shown here. Hence, the lightweight construction laws valid for bones are also valid for leaves. This means for the leaves that they reach the required structural stability with a minimum use of material and, hence, are optimized.

\section{References}

[1] Mattheck, C., Kappel, R., Tesari, I. \& Kraft, O., In Seilen denken. Konstruktionspraxis, 9, pp. 26-29, 2005.

[2] Beitz, W. \& Grote, K.-H., Dubbel - Taschenbuch für den Maschinenbau, Springer Verlag: Berlin Heidelberg New York, 20, 2001.

[3] Mattheck, C. \& Kappel, R., Festgekrallt - Verzweigung als Schubkiller. Konstruktionspraxis, 3, pp. 18-19, 2005. 